\title{
Sentient Artefacts: Acquiring User's Context Through Daily Objects
}

\author{
Kaori Fujinami and Tatsuo Nakajima \\ Department of Computer Science, Waseda University, Tokyo Japan \\ \{fujinami, tatsuo\}@dcl.info.waseda.ac.jp
}

\begin{abstract}
In this paper, we describe an augmentation of everyday artefact called sentient artefact. A sentient artefact is expected to capture the user's specific context implicitly and naturally from its original usage since such an everyday artefact has inherent roles and functionalities. Therefore, a context-aware space is built incrementally using the specific contextual information. We show three types of everyday artefact augmentation, and propose a sensor selection framework that allows an artefact developer to systematically identify desirable sensors. Also, we discuss expectations and issues on the augmentation through prototyping.
\end{abstract}

\section{Introduction}

Ubiquitous computing is envisioning to provide an intelligent environment, however it has not been realized yet. We consider one of the reason is the installation cost. Enabling technologies so far, e.g. location sensing systems and vision-based recognition systems, require complex infrastructures embedded into our environment, which increase the deployment cost. Also, a new type of devices that requires a user to learn its usage might provide him/her with a cognitive burden.

To address these issues, we are working on augmenting daily objects with computing capabilities like sensors and actuators, which capture a user's situation, context, and provide him/her its awareness in a natural and implicit way. We call such a daily object a "sentient artefact". We use a sentient artefact as a daily object that has inherent functionalities as usual. At the same time, it detects its state-of-use and utilizes the information as an input to a system. For example, a sentient door and sentient chair are utilized as an ordinary door and chair respectively. However, a system perceives a user's presence and state, e.g. inside the room, sitting on the chair, etc., and changes its behavior according to the contextual information. We believe the sentient artefact approach allows a developer to build context-aware applications easily. Also, from a user's aspect, he/she can utilize a context-aware service implicitly and naturally through the interaction with various sentient artefacts. A sentient artefact is expected to play a key role in realizing a ubiquitous computing environment in a practical way.

We have been prototyping various types of sentient artefacts so far. In this paper, we describe experiences from three of them: a chair, mirror, and toothbrush. We have found that an ad-hoc selection of sensors that depends on a developer's intuition fails to extract proper contextual information. Therefore, 
we propose a conceptual framework that allows a sentient artefact developer to select appropriate sensors. The framework utilizes the usage of the artefact and the observable phenomenon through the interaction with a user.

The structure of the paper is as follows. Section 2 shows early prototyping of the three sentient artefacts. In Section 3, a sensor selection framework is proposed through the prototyping. In the discussion section(Section 4), we describe 1) a variety of contextual information beyond the original state-of-use, 2) design issues and our approach towards artefacts integration, 3) structured artefact's information for robust and portable application, and 4) assessment of our approach that employs low-level sensors and combination of sentient artefacts for person identification. In Section 5 , we examine existing work regarding augmenting daily objects and extracting context through complex sensing infrastructure. Finally, we conclude the paper with future directions in Section 6.

\section{Prototyping of Sentient Artefacts}

We have been augmenting various everyday objects continuously. Here, we introduce the following three typical artefacts.

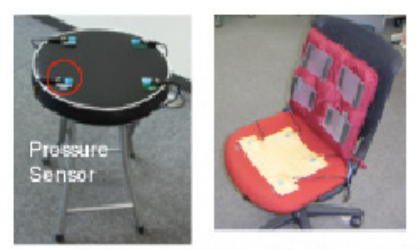

(a) Chair: Stool (left), office chair without (center) and with (right) an electronic compass.
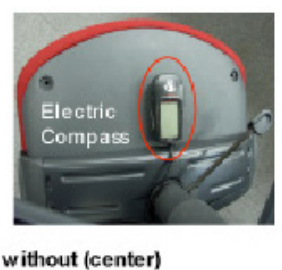

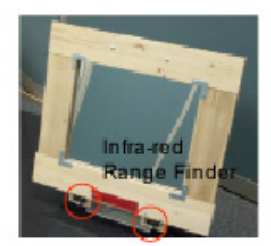

(b) AwareMirror

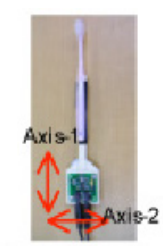

(c) Toothbrush

Fig. 1. Prototyped Artefacts

\subsection{Chair}

We use a chair for doing something with sitting on it. The activity at the chair is narrowed in conjunction with the type and/or location of the chair. For example, a chair in an office is utilized for supporting a user's work, while another chair in the kitchen is for eating or chatting. The change of states, i.e. from not sitting to sitting and vice versa, is a trigger for a system to invoke a specific service. We have developed the following three types of sentient chairs that can extract different types of contextual information:

1. Only an object's state, i.e. being put by something or not (Fig. 11.(a) left)

2. A person's sitting or not sitting with adverbial states (Fig. 1. (a) center)

3. An approximate direction of the face of a user (Fig. 1.(a) right)

The first case was built by simply augmenting a stool with four pressure sensors on the seat, where it does not distinguish a person from other objects. 
In the second case, an office chair with a back seat was adopted, and pressure sensors were attached properly so that the state of legs is extracted. Also, a photo sensor, a pressure sensor and a touch sensor were attached to detect the usage of the back seat. Finally, an office chair was augmented with an electric compass that detects terrestrial magnetism and specifies the direction of the back seat of the chair. These types of chairs suggest the need for an ontology of an artefact to provide applications with robustness and reliability, which will be discussed in more detail in Section 4.3 .

\subsection{Mirror}

AwareMirror (Fig. 1 (b)) is an augmented mirror that displays information relevant to a person in front of it on the periphery of his/her sights 4 . The augmentation is natural because we usually "use" a mirror to know our internal state, e.g. health, as well as external state, e.g. looks, through the reflection on the surface of it, which is easily extended to virtual features, e.g the schedule of the day, the weather forecasting to the destination. AwareMirror suggests an ideal interaction between a person and a computer embedded into our daily lives.

The AwareMirror project have also suggested socially acceptable sensing technology for the artefact augmentation. The state-of-use of a mirror should be the situation that the user is looking at the figure in the mirror. However, we have considered the state as the fact of detecting something in front of a mirror, which is realized by two infra-red range finders to remove a feeling of privacy violation. It is combined with the detection of utilization of a co-located toothbrush to extract more meaningful and accurate information.

\subsection{Toothbrush}

We have also got a survey result that people do not want to share a toothbrush with others, which means that the usage of a toothbrush strongly suggests that its owner is brushing his/her teeth. We have augmented a toothbrush with a two-axis accelerometer to detect the start, end, and the approximate number of brushing (Fig. 1 1 (c)) [5]. Also in [5], we have utilized a sentient toothbrush as an exclusive activity information source against the user's "sleeping", which means a sentient artefact is a building block for various applications, rather than a specific application.

\section{Sensor Selection Framework}

The sensors utilized in the prototyping were selected after some trials on a variety of sensors, which depends on a user's intuition. Such an ad-hoc way prevents an application developer from selecting appropriate sensors rapidly and consistently. In this section, we describe a framework to select suitable sensors for respective sentient artefacts in a systematic way. In our framework, the relationship between a user or his/her belonging and an artefact to augment is a key to find an appropriate phenomenon for sensing. The discussions in existing work [3, 6] show the 
catalogues of sensors that is utilized to specify a sensor from the phenomena to be measured. However, it lacks of earlier stages in terms of selecting appropriate sensors for artefact augmentation, i.e. what kind of state can be extracted, what kind of interaction is remarkable for the context extraction, what kind of phenomenon can be observed from the interaction, etc. Namely, our framework provides a sentient artefact developer with a systematic way to finally answer a question like "What kind of phenomenon is remarkable to extract the state-of-use of the target artefact most accurately?". The framework consists of five steps:

STEP1: Specify the state-of-use that a developer wishes to extract

STEP2: Analyze the usage of a target artefact

STEP3: Clarify the observable phenomena in use

STEP4: List the candidates of sensors

STEP5: Select the most preferable one from various aspects

In the following sections, we describe each step in more detail based on the prototyping results.

\subsection{Specify the State-of-Use to Extract (STEP1)}

This step is to answer the basic and essential question like "What state-of-use information is required?". This question can be separated into two detail questions: the level of abstraction and type of information. As depicted in Fig. 2, an office chair can offer two types of usage: the usage as a chair in nature (left side) and that is specific to a chair with back seat (right side). Also, each type might have more detailed usage, e.g. simply putting something on (the presence of an object), let a person sit on (the presence of a person), and let a person relaxed with his/her back lean against the back seat (the way of sitting). Therefore, first of all, a developer has to clarify his/her requirements for the extraction. However, they should be assessed whether the target artefact provides required state-of-use because they might not be provided by the artefacts functionalities.

\subsection{Analyze the Usage of Artefact (STEP2)}

The next step is to analyze the specified usage to answer the question "How to use it? or How to interact with it?". The result of the analysis classifies the usage into primitives, which include putting/removing, touching/leaving, pushing/pulling, rotating, shaking, approaching/leaving, stroing/extracting, etc. For example, in the case of sitting on a stool depicted in Fig. 1 1 (a)-left, a user's hip is "put" on the seat with some force, while a person sitting on an office chair (Fig. 1f(a)center/right) can "rotate" it and lean on the back seat, i.e. "touching".

Moreover, it is important to identify the ease of changing of the relationship, that may affect the weight of responsibility of the sensor. In the case of the above office chair, the relationship between a person and the back seat can be changed frequently since he/she might bend and lean, while the relationship between a person and the seat do hardly change. Therefore, to extract the presence of a person, one or more sensors should be attached primarily on the seat, and those of the back seat should be provided to supplement the primary ones. 


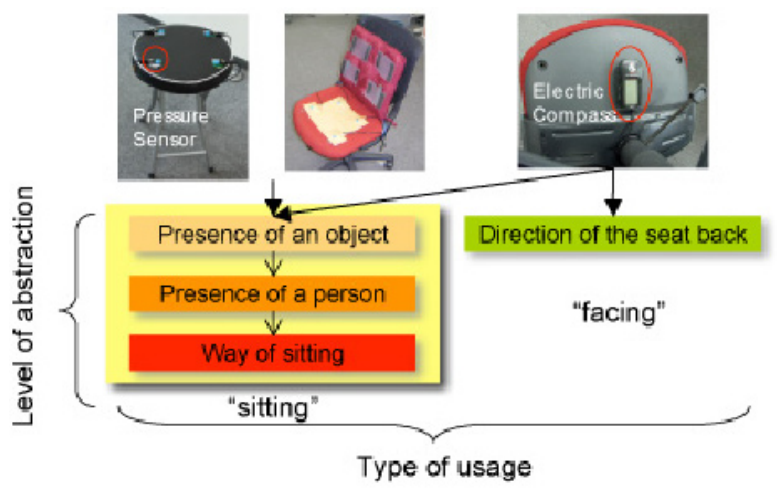

Fig. 2. Heterogeneity of the level of abstraction and type in daily object

\subsection{Clarify Observable Phenomena (STEP3)}

The third step is to clarify the observable phenomena against the primitives. For example, when something is "put" on the surface, there might be physical phenomena like the change of pressure on the surface, the vibration of the surface, making noise, the change of temperature on the surface (in case of a creature), the change of electric capacitance, etc. Although almost the same phenomena can be observed in the case of "touching" on the back seat of a chair, it is difficult to detect the change of temperature because the "touching" happens so often while the speed of changing temperature is slow. This means that "leaving" from the back seat might happen before a temperature reaches at a dedicated level. By the end of this step, the phenomena that contributes to extract the specified state-of-use become clear.

\subsection{List the Candidates of Sensors (STEP4)}

Hereafter, there is the literature to know how to sense a specific phenomenon, e.g. 3, 6]. Namely, the answer to the question like "What sensor can measure a change of force?" is easily found. There may be more than one sensing technology for each phenomenon, which will be identified in the final step.

\subsection{Select the most Preferable Sets of Sensor (STEP5)}

This is the final stage of the framework, where the most preferable sets of sensors are identified from many aspects, e.g. qualities, performance, form factor, cost, power consumption, availability, aesthetics, etc. The trade-offs needs to be resolved based on overall requirements for the prototyping or product.

\subsection{An Example to Use the Framework}

Figure 3 illustrates a part of the framework utilization, that represents the selection flow for a person's presence on an office chair, i.e. sitting on a chair. In SETP1, 


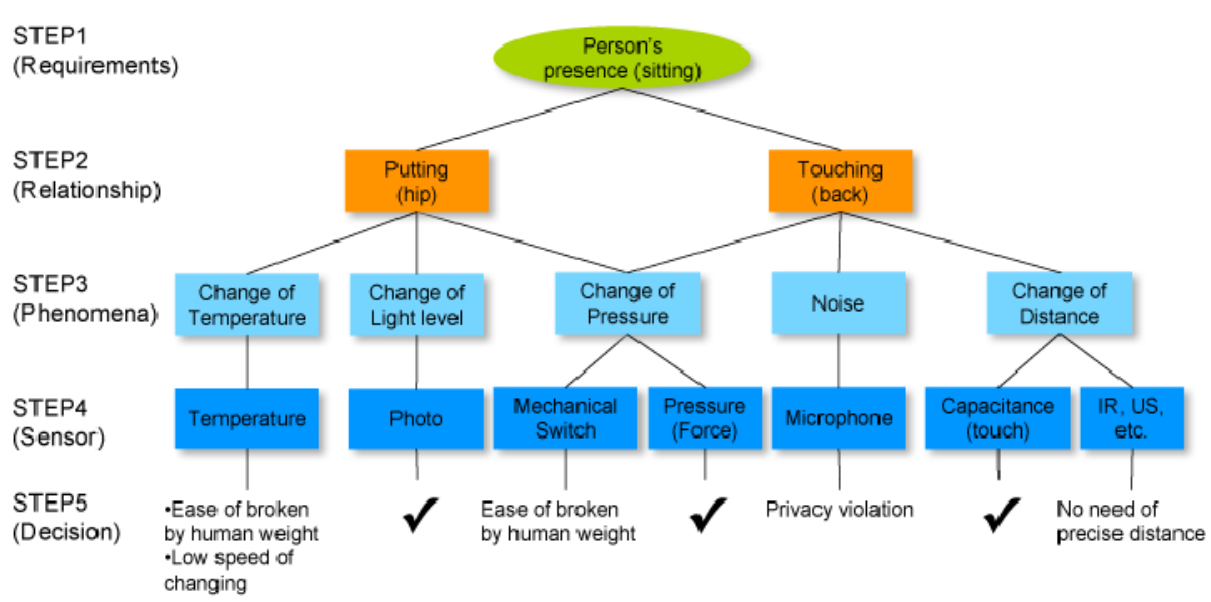

Fig. 3. An example of the framework usage (for person's presence on an office chair)

it begins with the state-of-use of interest, i.e. "presence", which can be divided into two relationship: 1) "putting" his/her hip on the seat and 2) "touching" his/her back to the back seat in STEP2. Then, in STEP3, five types of phenomena are identified, and then in STEP4, seven types of sensors are extracted based on the phenomena. Finally, in STEP5, a photo sensor and force sensor are selected for "hip-on-the-seat" detection, while a force sensor and touch sensor are chosen for "back-touching detection". To enhance the reliability of the sensing, more than two same sensors are to be utilized. A temperature sensor, mechanical switch, microphone, and IR (infra-red)/US (ultrasonic) sensors are rejected due to the ease of broken by human weight, low response speed, and/or privacy violation.

Our proposal is not the entire structure of a selection flow, instead the five steps that systematically identify sensors for the state-of-use extraction. The selection flow should be improved by the evaluation after prototyping, and also it should be extended incrementally through the development. Sharing the selection flow with others allows an artefact developer to follow the same way as successful development so that he/she becomes free from the ad-hoc selection.

\section{Discussion}

\subsection{Information Beyond State-of-Use}

Sentient artefacts provide various kinds of information that is used as contextual information beyond the initial state-of-use as described below.

Identity of Interacting Entity: An artefact interacts with a human (user) and another artefact. In case of the interaction with a human, a personal artefact identifies a user with high accuracy because it is assumed that the user is equals to the owner. So, the state-of-use is a trigger for a personalized service like the 
AwareMirror system [4]. An artefact also interacts with other artefacts, e.g. a cup on a desk. If the desk has an RFID reader, it can identify what is on it.

Location of a User: A stationary artefact like a door, mirror stand, etc. is the one that does not move. The characteristic allows a system to infer a user's location that offer the context with additional meaning. For example, a user in front of a mirror in the entrance might be in the different situation from who stands in front of a mirror in the washroom. The user's location is determined without any precise location sensing system if the location of the sentient artefact is known in some ways, e.g. manually specified.

Adverbial Information of State-of-Use: Adverbial context represents a user's context more richly, which can separate into two types: 1) an absolute and 2) relative adverbial context. Adverbial context with absolute values does not depend on a situation like a user's feeling and an application's requirements. In the statement "a user is sitting on a chair with his/her back touching on the chair's back once per minute", the italic part is the adverbial expression while "10 times per second" represents the user's situation of sitting absolutely. So, an application developer flexibly interprets it based on application requirements. However, an issue here is the selection of appropriate metrics, e.g. the frequency, average value, etc. On the other hand, the relative expression like "slowly", "suddenly", "angrily" is difficult to model at the development because such an expression is subjective. This is important because a sentient artefact should be a generic component for applications rather than an application specific one.

\subsection{Spatially Distributed Artefacts Integration}

A single sentient artefact provides a piece of a user's context. However, it is insufficient to describe more complex context in a robust way. So, sentient artefacts should communicate with external entities.

Communication Styles: The communication between an artefact and application is achieved in three ways: 1) event notification, 2) periodic transmission, and 3) retrieval from outside. Figure 4 illustrates the relationship among state, event, and also adverbial information. An event is generated as a result of an action performed by a user, e.g. sit down and stand up, while a state is represented by a period between events. In the figure, the state "sitting" represents from the event "sit down" to the other one "stand up". Adverbial information is attached to both an event and state (the rounded rectangle in the figure).

The event notification style is popular because many context-aware applications adapt their behavior according to the change of context. Also, it can minimize the communication. The periodic transmission is appropriate for sending monitored states continuously. However, this kind of communication should be minimized because it wastes the power of the artefact as well as bandwidth. The transmission should be invoked after detecting the start of original functionality of an artefact, e.g. "sitting", in order to avoid useless communication. This 
is an important aspect for an everyday artefact that has long idle time. States can also be provided by retrieval from outside entity, where the entity can get information at anytime. The direction of the back seat and the user's leg state, e.g. the left foot on the right, have been obtained in this way.

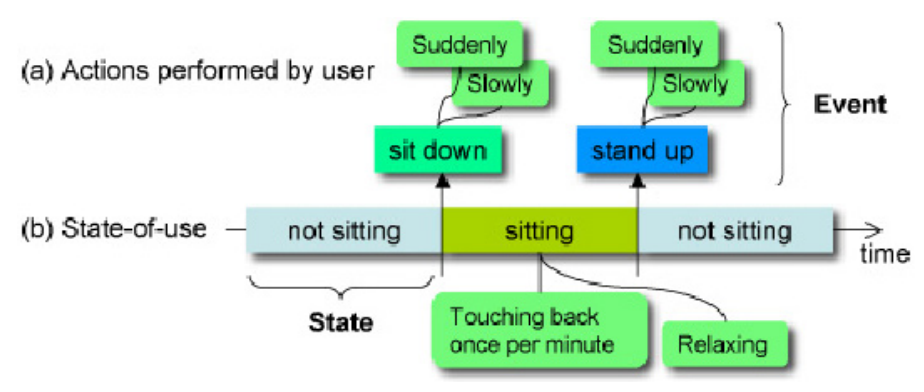

Fig. 4. Relationship among State, Event, and Adverbial Information

Centralized vs. Decentralized Architecture: We have developed an artefact integration middleware, Bazaar, which manages a model of world and provides a unified access to it [5]. Bazaar employs a centralized server architecture, where each artefact simply communicates with a server and application logics run. On the other hand, a decentralized peer-to-peer architecture has no central server and seems to be suitable for the artefacts communication because of scalability in the number of artefacts and the absence of single point of failure.

However, the application logic requires extraction of higher level context from each state-of-use. Also, it controls the presentation of context-awareness. We consider it is hard for a sentient artefact to execute these processes on behalf of others because it usually has limited resources. We expect a personal devices like a cellular phone and PDA to be a central server because they have enough resources. A user of the device is assumed to be its owner, which can identify the user and allows the device to provide a personalized service easily. Moreover, due to its local processing, privacy sensitive information does not reveal at all.

\subsection{Towards Robust and Portable Application}

A type of sentient artefact can vary in the level of abstraction that it perceives. As can be seen in Fig. 2 the sentient chairs shows various level of information abstraction, i.e. just presence of an object, and the way of sitting. The one with electronic compass also provides the direction of the seat back, a type of usage "facing", in addition to the original one, "sitting". If an application requires information of just presence of an object, all the three types of chairs can be utilized. This means these chairs are replaceable with each other at the place, and that the application running at one space is portable to another. Therefore, the state-of-use of chairs should be properly structured, that is, an ontology needs 
to be specified. Moreover, an ontology for much wider class like an "artefact" than a single class "chair" allows an application to replace a chair with a door, for example, as an artefact that provides information of the user's presence.

Furthermore, to infer a user's context accurately and thus to build robust applications, the quality of information like freshness, accuracy, and confidence should be considered as well as the information contents. We need to identify suitable ones and include them into a context model as meta-information, which allows an application developer to implement appropriate adaptation strategies.

\subsection{Privacy Concerns}

One of our design principles is augmenting artefacts with low-level sensing technologies, e.g. accelerometer, infra-red range finder, etc. This is because the user's feelings of privacy violation. A video camera and microphone can be utilized to detect rich context like the user's activity, identity, location, and even emotion and intension without the user's explicit input. However, our user survey at the AwareMirror development has revealed the testees' obtrusive feelings of being watched even though they know the benefits of the method 4 .

In terms of user identification, the testees pointed out that the merit of using a sentient artefact that is hardly shared with others is its implicity. As described in Section 2.2, it is utilized to start showing personalized information on the mirror without a user's explicit interaction with the mirror. However, the possibilities of intentional or unintentional use by other persons were also pointed. We consider users know the trade-off between the relevance of information and the efforts they need to make to keep it secret. So, explicit identification method like fingerprint recognition should be accepted if the system provides a highly relevant information like credit card number. Furthermore, if the toothbrush is utilized in a closed reliable group like a family, these issues are suppressed. Therefore a system should separate the contents utilization, e.g. using the identity, from the acquisition method, e.g. sentient artefact-based, biometric-based, etc., for the method's flexible selection based on the application requirements.

\section{Related Work}

Regarding to the deployment of smart environments, Sentient Computing [1] proposed a location sensing system that utilizes the ultrasonic and radio frequency signal [8. To measure the position of an object within a cubic inch, it requires dense ultrasonic transceivers, which is impossible to deploy and maintain without special cares. Moreover, the system provides only location context, which means a system's awareness of a user's context is limited. However, a sentient artefact provides its user's state-of-use as a primary context of the user, so the information source is closer to the user, which is considered to be more accurate and meaningful to him/her. And, as described in Section 4.1, it can provide various types of information. The more the type of artefacts increase, the more the kinds of extracted context increases. Therefore, neither precise nor dense location sensing system is required. 
The MediaCups project [2] and its succeeding project of SmartITs [7] provide insights into the augmentation of artefacts with sensing and proecssing. The notion of artefacts computing composed of sensor augmented artefact provides a mean to obtaining human context implicitly, which has been greatly influenced the notion of sentient artefact. We are working on representing an artefact formally and integrating them systematically, which must be applicable to sentient artefacts based on the SmartITs platform.

\section{Conclusions and Future Direction}

A sentient artefact is designed to perceive its usage as a user's context. We have introduced three artefacts' prototyping experiences, and proposed a conceptual framework that systematically identifies appropriate sensors for specific stateof-use of an artefact. The following discussions are presented 1) the information expected through the state-of-use of sentient artefact, 2) communication styles and architectural comparison towards sentient artefacts integration, 3) needs for the ontology development and meta-information definition for robust and portable application, and 4) the sensing technologies to capture context considering privacy aspects.

The sentient artefact-based context acquisition is expected to require less precise or no location system, and to provide accurate information than just touching an artefact, however, we need to assess them through practical application developments and user evaluation. Also, we are working on developing an artefact ontology including meta-information.

\section{References}

1. M. Addlesee, R. Curwen, S. Hodges, J. Newman, A. Ward, and A. Hopper. Implementing a Sentient Computing System. IEEE Computer Society, pages 50-56, Aug. 2001.

2. M. Beigl, H.-W. Gellersen, and A. Schmidt. MediaCups: Experience with Design and Use of Computer-Augmented Everyday Objects. Computer Networks, Special Issue on Pervasive Computing, 35(4):401-409, March 2001.

3. M. Beigl, A. Krohn, T. Zimmer, and C. Decker. Typical Sensors needed in Ubiquitous and Pervasive Computing. In Proceedings of the First International Workshop on Networked Sensing Systems (INSS) 2004, pages 153-158, June 2004.

4. K. Fujinami, F. Kawsar, and T. Nakajima. AwareMirror: A Personalized Display using a Mirror. In Proceedings of International Conference on Pervasive Computing, Pervasive2005, LNCS 3468, pages 315-332, May 2005.

5. K. Fujinami and T. Nakajima. Towards System Software for Physical Space Applications. In Proceedings of ACM Symposium on Applied Computing(SAC) 2005, pages 1613-1620, March 2005.

6. A. Schmidt and K. V. Laerhoven. How to build smart appliances. IEEE Personal Communications, pages $66-71,2001$.

7. The Smart-ITs project. The smart-its. URL: <http://www.smart-its.org/>.

8. A. Ward, A. Jones, and A. Hopper. A New Location Technique for the Active Office. IEEE Personnel Communications, 4(5):42-47, October 1997. 